\title{
Glycosylation of the HIV-1 Env V1V2 loop to form a native-like structure may not be essential with a nanoparticle vaccine
}

\author{
Christopher P Karch ${ }^{1,2}$, Gary R Matyas ${ }^{1}$, Peter Burkhard ${ }^{3}$ \& Zoltan Beck*,1,2 \\ ${ }^{1}$ U.S. Military HIV Research Program, Walter Reed Army Institute of Research, 503 Robert Grant Ave, Silver Spring, MD 20910, USA \\ ${ }^{2}$ Henry M. Jackson Foundation for the Advancement of Military Medicine, 6720A Rockledge Drive, Bethesda, Maryland 20817. \\ USA \\ ${ }^{3}$ Alpha-O Peptides, Lörracherstrasse 50, 4125 Riehen, Switzerland \\ *Author for correspondence: Tel.: +1 301319 2290; zbeck@hivresearch.org
}

“'vaccination with unglycosylated HIV-1 Env V1V2 antigens that fold into native-like confirmation may generate functional antibodies at higher titers with higher affinity, ideally leading to a better vaccine candidate"

First draft submitted: 18 October 2018; Accepted for publication: 20 December 2018; Published online: 10 January 2019

\section{Glycosylation for viral proteins}

Glycosylation of viral proteins plays an important role in the lifecycle of the virus. The presence of glycans on viral proteins aids in correct folding and maturation. Glycosylation has also been shown to allow for the release of infectious progeny viral particles and is essential in the interaction with host receptors, leading to binding and eventually infection. Glycans can also mask immunodominant epitopes on the viral surface; protecting the virus from the host immune system. In the host, the virus uses cellular glycosylation machinery to 'camouflage' itself, however, in some instances, the unique virus-specific glycosylation pattern can lead to new targets for the immune system [1].

\section{Importance of glycosylation for vaccine development against enveloped viruses}

A major consideration for subunit vaccine development is the native-like presentation of the antigen of interest. Out of the many possible immunogens of enveloped viruses, one of the strongest targets is the membrane fusion protein. These integral membrane glycoproteins facilitate fusion of the virus with the host cell's plasma membrane, and therefore are essential for the productive infection [2,3]. Antibodies raised to fusion proteins can either directly prevent the initial viral infection of cells, or lead to the death of infected cells by different cellular mechanisms [4]. Epitopes of these fusion proteins in their native-like conformation may elicit functional antibodies that recognize the pathogen upon subsequent natural exposure [5]. Glycosylation plays a very contradictory role in this regard; on one hand it is generally important for protein folding, however, on the other hand it can occlude epitopes reducing the immune response [1]. In many cases, subunit vaccines are produced in mammalian cells that have posttranslational modification machinery; however, the glycosylation pattern may be different from the native virus [6].

A well-studied example of an enveloped viral fusion protein is hemagglutinin, from the influenza virus. It consists of a variable immunodominant head domain and a conserved stem domain [7]. Expression of a full-length hemagglutinin with the correct quaternary structure requires mammalian cells. However, parts of hemagglutinin produced in E. coli can also fold spontaneously, and the correct conformation can be facilitated by a carrier [8-15]. These unglycosylated antigens can be designed and displayed on a nanoparticle such that they are presented in a native-like trimeric form, increasing the immunogenicity of these vaccine candidates [9,11-15]. Many of these vaccines have been shown to be efficacious in both animal models and human clinical trials, indicating that the correct structure of the antigen, even without the presence of glycans, is essential for the development of an effective immune response [16-18].

Future Medicine 


\section{Glycosylation of HIV-1 envelope protein}

The only surface protein of human immunodeficiency virus-1 (HIV-1) is the Env fusion protein. Env is heavily glycosylated, with glycans composing up to half of its mass [19]. During maturation of Env, the full-length gp160 is cleaved by a protease into gp 41 and gp120, which remain associated forming Env heterodimers. Three heterodimers form a trimeric spike, and approximately 14 spikes decorate the surface of the virus [20,21]. The majority, about $62-79 \%$ of the gp120 glycans present on virus, are underprocessed N-linked high mannose, because the high density of the glycosylation sites can prevent the further processing steps of the glycans by the enzymes in the Golgi apparatus [22]. Changes in the glycosylation pattern of gp120 can alter the receptor binding and infection by the virus, as well as the susceptibility to antibodies [23,24]. In addition, the high mutation rate of HIV-1 results in an inconsistent level of glycosylation. For example, transmitter/founder viruses generally have a lower number of $\mathrm{N}$-linked glycosylation sites than chronic viruses and appear to be more infectious [25].

A major effort has been undertaken to understand the role of glycans in respect to broadly neutralizing antibodies (bnAbs) to the Env protein of HIV-1 [26]. Some of these antibodies directly interact with glycans. A well-known bnAb that exclusively recognizes the glycosylation pattern is $2 \mathrm{G} 12$ [23]. Most of the bnAbs interacting with both the glycan and the amino acid sequence of gp120 have structural features, such as long CDRH3 loops, to penetrate the glycan shield [27]. Binding to the virus and neutralization by many of these bnAbs primarily depend on the quaternary structure of the protein, which can, however, be affected by the presence of the glycans. Between 10 and $30 \%$ of people infected with HIV-1 develop bnAbs with cross-clade neutralizing activity within 3 years of infection but while controlling the infection, it does not result in sterilizing immunity [28]. Considering that only a small fraction of antibodies to HIV-1 elicit broadly neutralizing function, the low concentration of these bnAbs in vivo, and their late occurrence during disease progression; a potentially different approach to generate bnAbs for an effective prophylactic vaccine may be necessary [26. It raises the question: is it possible to induce high titer bnAbs with high binding affinity to the virus with an Env immunogen that is not glycosylated, but maintains the conformation of the protein?

\section{Vaccine development with nanoparticle-displayed HIV-1 V1V2 trimers}

One of the most important regions of the HIV-1 Env protein is the V1V2 loop. IgG antibodies raised against this loop were inversely correlated with the risk of infection in the RV144 clinical trial [29]. Studies indicate that the V1V2 loop of the protein does not assume a unique defined structure [30]. As a result of the high density of glycans on the Env protein, most efforts in subunit vaccine development have used mammalian cells for bio-production. However, similar to the influenza hemagglutinin, a potential HIV-1 V1V2 vaccine candidate could be developed with an antigen that is expressed in E. coli, without glycosylation but with native-like conformation to induce high titers of high affinity antibodies that could prevent the initial infection [31].

The use of a carrier may be essential to the development of a successful V1V2 vaccine candidate. One such technology is the self-assembling protein nanoparticle (SAPNs). SAPNs are built on coiled-coil protein folding domains, which are composed of two or more $\alpha$-helices. Glycosylation of the HIV-1 Env V1V2 loop to form a native-like structure may not be essential with a nanoparticle vaccine [32]. By choosing the correct combination of coiled-coil domains, protein folding can be optimized and the antigen of interest can be folded into a native-like conformation. This technology has been successfully applied in the development of influenza, SARS, toxoplasma and malaria vaccine candidates, in all of which the immunogens were unglycosylated [9,33-36].

Although the importance of glycosylation for viral maturation and folding of envelope proteins is clear, it remains unclear if proper glycosylation or some level of glycosylation of the immunogen is necessary for inducing protective immune response by an HIV-1 vaccine. Some attempts have been carried out to correlate the glycosylation status of the HIV-1 antigen and the immune response [37-39]. It is hypothesized that for protective immune response to HIV-1, generation of conformational antibodies is required. These antibodies can be generated only by properly folded immunogens that have either the native glycosylation pattern or are displayed on a nanoparticle carrier to enable the native-like trimeric antigen conformation. Producing subunit vaccine candidates in mammalian cells to generate glycosylation is difficult; the yield tends to be lower, and cost tends to be higher. The glycosylation pattern obtained in expression systems may be different than those present on the virion, ultimately affecting the structure of the antigen [40]. By using the SAPN technology, we were able to produce a vaccine candidate in $E$. coli capable of raising high titers of antibodies to the V1V2 loop. The structure of the antigen was determined to be native-like by the binding to known conformational V1V2 antibodies such as PG9, PG16 and PGT145 [31]. We hypothesize that vaccination with unglycosylated HIV-1 Env V1V2 antigens that fold into native-like confirmation 
may generate functional antibodies at higher titers with higher affinity, ideally leading to a better vaccine candidate. To validate this hypothesis, further studies are required to compare the functionality of antibodies from animals vaccinated with unglycosylated and glycosylated SAPN immunogens. A prime boost strategy with a vaccination using unglycosylated immunogen followed by a glycosylated immunogen can be used in an attempt to generate high titers of anti-V1V2 antibodies that recognize the virion, regardless of the glycosylation status.

\section{Financial \& competing interests disclosure}

The views expressed are those of the authors and should not be construed to represent the positions of the US Army or the Department of Defense. This work was supported by a cooperative agreement (W81XWH-11-2-0174) between the Henry M Jackson Foundation for the Advancement of Military Medicine, Inc., and the US Department of Defense. The work was further supported by an Avant Garde award to GRM from the National Institute on Drug Abuse, NIH (grant number 1DP1DA034787-01). P Burkhard has an interest in the company Alpha-O Peptides that has patents or patents pending on the technology. The authors have no other relevant affiliations or financial involvement with any organization or entity with a financial interest in or financial conflict with the subject matter or materials discussed in the manuscript apart from those disclosed.

No writing assistance was utilized in the production of this manuscript.

\section{Open access}

This work is licensed under the Attribution-NonCommercial-NoDerivatives 4.0 Unported License. To view a copy of this license, visit http://creativecommons.org/licenses/by-nc-nd/4.0/

\section{References}

1. Bagdonaite I, Wandall HH. Global aspects of viral glycosylation. Glycobiology 28(7), 443-467 (2018).

2. Cohen FS. How viruses invade cells. Biophys. J. 110(5), 1028-1032 (2016).

3. Rey FA, Lok SM. Common features of enveloped viruses and implications for immunogen design for next-generation vaccines. Cell 172(6), 1319-1334 (2018).

4. Lu LL, Suscovich TJ, Fortune SM, Alter G. Beyond binding: antibody effector functions in infectious diseases. Nat. Rev. Immunol. 18(1), 46-61 (2018).

5. Koellhoffer JF, Higgins CD, Lai JR. Protein engineering strategies for the development of viral vaccines and immunotherapeutics. FEBS Lett. 588(2), 298-307 (2014).

6. Legastelois I, Buffin S, Peubez I, Mignon C, Sodoyer R, Werle B. Non-conventional expression systems for the production of vaccine proteins and immunotherapeutic molecules. Hum. Vaccin. Immunother. 13(4), 947-961 (2017).

7. Russell CJ, Hu M, Okda FA. Influenza hemagglutinin protein stability, activation, and pandemic risk. Trends Microbiol. 26(10), 841-853 (2018).

8. Roberts PC, Garten W, Klenk HD. Role of conserved glycosylation sites in maturation and transport of influenza A virus hemagglutinin. J. Virol. 67(6), 3048-3060 (1993).

9. Karch CP, Li J, Kulangara C et al. Vaccination with self-adjuvanted protein nanoparticles provides protection against lethal influenza challenge. Nanomedicine 13(1), 241-251 (2017).

10. Liu G, Tarbet B, Song L et al. Immunogenicity and efficacy of flagellin-fused vaccine candidates targeting 2009 pandemic H1N1 influenza in mice. PLoS ONE 6(6), e20928 (2011).

11. Jegerlehner A, Zabel F, Langer A et al. Bacterially produced recombinant influenza vaccines based on virus-like particles. PLoS ONE 8(11), e78947 (2013).

12. Verma S, Dimitrova M, Munjal A et al. Oligomeric recombinant $\mathrm{H} 5 \mathrm{HA} 1$ vaccine produced in bacteria protects ferrets from homologous and heterologous wild-type $\mathrm{H} 5 \mathrm{~N} 1$ influenza challenge and controls viral loads better than subunit $\mathrm{H} 5 \mathrm{~N} 1$ vaccine by eliciting high-affinity antibodies. J. Virol. 86(22), 12283-12293 (2012).

13. Khurana $\mathrm{S}$, Verma $\mathrm{S}$, Verma $\mathrm{N}$ et al. Bacterial $\mathrm{HA1}$ vaccine against pandemic $\mathrm{H} 5 \mathrm{~N} 1$ influenza virus: evidence of oligomerization, hemagglutination, and cross-protective immunity in ferrets. J. Virol. 85(3), 1246-1256 (2011).

14. Khurana S, Verma S, Verma N et al. Properly folded bacterially expressed H1N1 hemagglutinin globular head and ectodomain vaccines protect ferrets against H1N1 pandemic influenza virus. PLoS ONE 5(7), e11548 (2010).

15. Khurana S, Larkin C, Verma $S$ et al. Recombinant HA1 produced in E. coli forms functional oligomers and generates strain-specific SRID potency antibodies for pandemic influenza vaccines. Vaccine 29(34), 5657-5665 (2011).

16. Treanor JJ, Taylor DN, Tussey L et al. Safety and immunogenicity of a recombinant hemagglutinin influenza-flagellin fusion vaccine (VAX125) in healthy young adults. Vaccine 28(52), 8268-8274 (2010).

17. Taylor DN, Treanor JJ, Strout C et al. Induction of a potent immune response in the elderly using the TLR-5 agonist, flagellin, with a recombinant hemagglutinin influenza-flagellin fusion vaccine (VAX125, STF2.HA1 SI). Vaccine 29(31), 4897-4902 (2011). 
18. Taylor DN, Treanor JJ, Sheldon EA et al. Development of VAX128, a recombinant hemagglutinin (HA) influenza-flagellin fusion vaccine with improved safety and immune response. Vaccine 30(39), 5761-5769 (2012).

19. Leonard CK, Spellman MW, Riddle L, Harris RJ, Thomas JN, Gregory TJ. Assignment of intrachain disulfide bonds and characterization of potential glycosylation sites of the type 1 recombinant human immunodeficiency virus envelope glycoprotein (gp120) expressed in Chinese hamster ovary cells. J. Biol. Chem. 265(18), 10373-10382 (1990).

20. Behrens AJ, Crispin M. Structural principles controlling HIV envelope glycosylation. Curr. Opin. Struct. Biol. 44, 125-133 (2017).

21. Schiller J, Chackerian B. Why HIV virions have low numbers of envelope spikes: implications for vaccine development. PLoS Pathog. 10(8), e1004254 (2014).

22. Bonomelli C, Doores KJ, Dunlop DC et al. The glycan shield of HIV is predominantly oligomannose independently of production system or viral clade. PLoS ONE 6(8), e23521 (2011).

23. Crispin M, Ward AB, Wilson IA. Structure and immune recognition of the HIV glycan shield. Annu. Rev. Biophys. doi:10.1146/annurev-biophys-060414-034156 (2018) (Epub ahead of print).

24. Huang X, Jin W, Hu K et al. Highly conserved HIV-1 gp120 glycans proximal to CD4-binding region affect viral infectivity and neutralizing antibody induction. Virology 423(1), 97-106 (2012).

25. Parrish NF, Gao F, Li H et al. Phenotypic properties of transmitted founder HIV-1. Proc. Natl Acad. Sci. USA 110(17), 6626-6633 (2013).

26. Kwong PD, Mascola JR, Nabel GJ. Broadly neutralizing antibodies and the search for an HIV-1 vaccine: the end of the beginning. Nat. Rev. Immunol. 13(9), 693-701 (2013).

27. Crispin M, Bowden TA. Antibodies expose multiple weaknesses in the glycan shield of HIV. Nat. Struct. Mol. Biol. 20(7), 771-772 (2013).

28. Landais E, Moore PL. Development of broadly neutralizing antibodies in HIV-1 infected elite neutralizers. Retrovirology 15(1), 61 (2018).

29. Haynes BF, Gilbert PB, Mcelrath MJ et al. Immune-correlates analysis of an HIV-1 vaccine efficacy trial. N. Engl. J. Med. 366(14), 1275-1286 (2012).

30. O’ Connell RJ, Kim JH, Excler JL. The HIV-1 gp120 V1V2 loop: structure, function and importance for vaccine development. Expert Rev. Vaccines 13(12), 1489-1500 (2014).

31. Karch CP, Bai H, Torres OB et al. Design and characterization of a self-assembling protein nanoparticle displaying HIV-1 Env V1 V2 loop in a native-like trimeric conformation as vaccine antigen. Nanomedicine doi:10.1016/j.nano.2018.12.001 (2018) (Epub ahead of print).

32. Karch CP, Matyas GR, Burkhard P, Beck Z. Self-assembling protein nanoparticles: implications for HIV-1 vaccine development. Nanomedicine 13(17), 2121-2125 (2018).

33. Karch CP, Doll T, Paulillo SM et al. The use of a P. falciparum specific coiled-coil domain to construct a self-assembling protein nanoparticle vaccine to prevent malaria. J. Nanobiotechnol. 15(1), 62 (2017).

34. Kaba SA, Karch CP, Seth L et al. Self-assembling protein nanoparticles with built-in flagellin domains increases protective efficacy of a Plasmodium falciparum based vaccine. Vaccine 36(6), 906-914 (2018).

35. El Bissati K, Zhou Y, Paulillo SM et al. Protein nanovaccine confers robust immunity against toxoplasma. NPJ Vacc. 2, 24 (2017).

36. Pimentel TA, Yan Z, Jeffers SA, Holmes KV, Hodges RS, Burkhard P. Peptide nanoparticles as novel immunogens: design and analysis of a prototypic severe acute respiratory syndrome vaccine. Chem. Biol. Drug Des. 73(1), 53-61 (2009).

37. Zhou T, Doria-Rose NA, Cheng C et al. Quantification of the impact of the HIV-1-glycan shield on antibody elicitation. Cell Rep. 19(4), 719-732 (2017).

38. Mccoy LE, Van Gils MJ, Ozorowski G et al. Holes in the glycan shield of the native HIV envelope are a target of trimer-elicited neutralizing antibodies. Cell Rep. 16(9), 2327-2338 (2016).

39. Dubrovskaya V, Guenaga J, De Val N et al. Targeted N-glycan deletion at the receptor-binding site retains HIV Env NFL trimer integrity and accelerates the elicited antibody response. PLoS Pathog. 13(9), e1006614 (2017).

40. Fuenmayor J, Godia F, Cervera L. Production of virus-like particles for vaccines. N. Biotechnol. 39(Pt B), 174-180 (2017). 